\title{
Current Trends in Computational Geomechanics and Geotechnical Engineering
}

\author{
MB Can Ülker* \\ Istanbul Technical University, Turkey
}

*Corresponding author: MB Can Ülker, Istanbul Technical University, Earthquake Engineering and Disaster Management Institute, Istanbul, Turkey.

Received Date: October 09, 2018

Published Date: November 20, 2018

\begin{abstract}
Computational geomechanics is the field of computational mechanics where geo-engineering systems are analyzed using the principles of mechanics through numerical methods. In those systems lie porous media with a variety of multi-phase materials such as soils, rock, composites or living tissues. Geomechanics is the branch of mechanics in that response and instability of such porous materials are studied under external loadings such as earthquakes, waves, varying heads of flow during various drainage conditions etc. Among those effects, earthquakes constitute a significant part of the design of geo-engineering systems. Geotechnical considerations are particularly important in identifying the conditions leading to instability of such systems under seismic excitations. Here, seismic soil behaviors are necessary properties to be determined to decide whether there will be permanent damage in foundation soils or indirectly in the upper structures during earthquakes. Therefore, it is of utmost significance that earthquake-induced soil behavior is both measured in laboratory experiments and modeled through theoretical and numerical frameworks. While the former have been conducted since 1960's, it had to take about two more decades for latter works to emerge. As the geotechnical earthquake engineering is a common field of application of geomechanics, another such field is the geotechnical coastal engineering. Wave-induced seabed response is of major concern here to engineers and researchers particularly around coastal and offshore structures. From a broader perspective, it looks like the field of computational geomechanics has currently shifted to three main topics: i) Recent challenges of numerical analyses in geomechanics and geotechnical engineering, ii) Analysis of large deformation and post failure response in geotechnical engineering, iii) Modeling and minimizing uncertainty for design and monitoring in geotechnical engineering. For the first issue of our newborn Journal: this short paper briefly summarizes some recent examples of problems from the fields of geotechnical earthquake engineering and geotechnical coastal engineering.
\end{abstract}

Keywords: Coastal geotechnics; Earthquake; Earthquake geotechnics; Geomechanics; Modeling; Numerical methods; Waves

\section{Introduction}

Computational geomechanics deals with numerical analysis of response and instability of porous media and related structural systems. One can argue that in the case of soils, apart from the elemental level constitutive behavior, interaction of soil layers with structures built around them brings an additional source of complexity due to nonlinear response aspects. There are many sub-fields of geomechanics where such aspects should be handled with care. Geotechnical earthquake engineering and geotechnical coastal engineering are two of those most commonly encountered sub-fields. In the case of geotechnical earthquake engineering, the actual response of soils and soil-structure systems under earthquake shaking is frequency-dependent and elemental soil behaviors determine how much of induced energy will be absorbed by soils and how much of it will be transferred to the upper structures. In this short opinion, the aim is to focus on topics where accurate modeling of soil behavior is indispensable. It is important that one has the necessary understanding of cyclic soil plasticity and incorporates related theoretical models into their numerical algorithms to provide accurate solutions to the earthquake-related problems.

In the case of coastal and offshore engineering where the problems take place in ocean environments, wave loads become a major issue and hence wave-induced dynamic response and instability of seabed-structure systems are to be studied. As far as geo-engineering is concerned, problems in this subfield are typically related with energy and coastal protection. That is, energy structures; such as offshore wind-turbines, and embedded pipelines as well as coastal protection type structures; levees, breakwaters etc. Dynamic response and instability of seabed-structure systems are to be modeled against wave action. 


\section{Current Trends}

Currently the field of computational geomechanics deals with three main topics: i) Recent challenges of numerical analyses of geosystems, ii) Analysis of large deformation and post failure response of soils, iii) Modeling and minimizing uncertainty for design and monitoring in geomechanics and geotechnical engineering. Recent challenges of numerical analyses in the above-mentioned two subfields of geotechnical earthquake engineering and geotechnical coastal engineering are associated with how the soil is modeled at elemental level under dynamic loading as well as how soil-structure interaction is handled numerically. Firstly, the physical model of the problem where the structural components are introduced into the soil media at this stage, is idealized as a simplified model whose response is governed by mathematical equations along with a set of prescribed boundary and initial conditions. These conditions are specified in terms of the field variables of the problem along with their spatial and temporal derivatives giving the forces exerted on the system. Such field variables are essentially the unknown degrees of freedom of the problem. These analysis steps are then ended with efficient and accurate solution of the governing equations using robust algorithms and computer power.

Another challenge is associated with analysis of large deformation and post failure response in geotechnical engineering. Large deformation can be observed in many geomechanics problems, particularly the ones that are related to soft ground movement. Deformation of sub-soils due to earthquake loading is one of typical examples. Another serious issue is the "post liquefaction behavior". That is, settlement and lateral spreading of loosely deposited saturated sand layers subjected to seismic excitations. An example for such challenges from the coastal environment is tsunami-induced mud flow and failures along coastlines. In the analysis of such problems, steps of numerical modeling mentioned above do not change. However, now there is another source of nonlinearity due to large deformations which need to be included in the computer codes.

Modeling and minimizing uncertainty for design and monitoring is another trendy 'hot topic' in geotechnical engineering. We, geotechnical engineers always suffer from lack of quality field and laboratory data to be used in our analyses and computations. Often times, we do not have access to accurate ranges of initial and boundary conditions, in particular, material properties and initial stress distributions. Inadequate and insufficient information in geotechnical problems frequently lead to a discrepancy between prediction and measurement as a result of a various degrees of uncertainties. Thus, in order to deal with such uncertainties from the lack of necessary information, a separate field of "stochastic geomechanics" or "probabilistic geotechnical engineering" has emerged over the last few decades. For better prediction of soil and structural behaviors, supplemental information on such conditions is vital. Data assimilation and inverse analysis are promising tools of the probabilistic approach for this purpose. The optimal values of the material properties, which are uncertain a-prior to the engineers, can be determined based upon detailed site investigation, essentially minimizing the gap between prediction and measurement. Stochastic analysis is a method that considers uncertainty as a probabilistic variable which is employed to assess the possibility of failure or other limit states of soil-structure system resulting in useful output data for performance-based design.

\section{Acknowledgment}

None.

\section{Conflict of Interest}

No conflict of interest. 